\title{
Numerical analysis of confined flow past permeable square cylinder with CE/SE method
}

Article in Progress in Computational Fluid Dynamics An International Journal · January 2015

CITATIONS

0

1 author:

Duoxing Yang

China Earthquake Administration

29 PUBLICATIONS 108 CITATIONS

SEE PROFILE
READS

86

Some of the authors of this publication are also working on these related projects:

Project

Enhanced heat transfer and energy conversion from deep geothermal reservoir based on nanofluid techniques View project

All content following this page was uploaded by Duoxing Yang on 25 November 2015. 


\title{
Numerical analysis of confined flow past permeable square cylinder with CEISE method
}

\section{Luhe Yang and Duoxing Yang*}

Institute of Crustal Dynamics, CEA, China

Email: yanglh@nankai.edu.cn

Email: dxyangcea@yahoo.com

Email: yangdx@mail.iggcas.ac.cn

*Corresponding author

\section{Lianzhong Zhang}

School of Physics, Nankai University, China

Email: zhanglz@nankai.edu.cn

\section{Deliang Zhang}

Institute of Mechanics,

CAS,

No.15 Beisihuanxi Road, Beijing, 100190, China

Email: dlzhang@imech.ac.cn

\section{Ziqiu Xue}

Research Institute of Innovative Technology for the Earth, 9-2, Kizugawadai, Kizugawa-Shi, Kyoto, 619-0292, Japan

Email: xue@rite.or.jp

\begin{abstract}
The unsteady flow field past a two-dimensional permeable square cylinder was predicted by an updated CE/SE method. The periodic patterns of streamline and vortex shedding were obtained. The evolution of lift and drag coefficients were analysed. Results of 2D computations were compared with the experimental data. It found that the Reynolds number dominated the magnitude and frequency of the vorticity given porosity and Darcy number. It proved that the CE/SE method was an effective tool for predicting viscous flows in porous media.
\end{abstract}

Keywords: porous media; unsteady flow; CE/SE method; computational fluid dynamics; CFD.

Reference to this paper should be made as follows: Yang, L., Yang, D., Zhang, L., Zhang, D. and Xue, Z. (xxxx) 'Numerical analysis of confined flow past permeable square cylinder with CE/SE method', Progress in Computational Fluid Dynamics, Vol. X, No. Y, pp.xxx-Xxx.

Biographical notes: Duoxing Yang is a Full Professor in the Institute of Crustal Dynamics, CEA. His research interests involve heat and mass transfer in porous media. He holds a $\mathrm{PhD}$ in Hydrology. He is an editorial board member of International Journal on Numerical and Analytical Method in Engineering (IRENA).

Luhe Yang is currently a candidate in the Master degree in the Institute of Crustal Dynamics, majoring in Geophysics.

Lianzhong Zhang is a Physics Professor in Nankai University. He obtained his Doctoral degree in Physics, doing research on theoretical physics and its applications.

Deliang Zhang is a Senior Professor in Institute of Mechanics, CAS, conducting computational fluid dynamics.

Ziqiu Xue is a Chief Researcher in the Research Institute of Innovative Technology for the Earth, specialising rock mechanics. He holds a PhD in Geophysics. 


\section{Introduction}

Fluid mechanics in porous media has been widely applied in hydro-geological investigation, underground oil recovery, agricultural irrigation technology and design of reservoir works ever since the last century. Subsequently, it is playing a more and more important role in many relevant fields of science in recent years, which has been applied in artificial materials such as glass fibre, activated charcoal, building materials and vascular networks in micro-organisms. Thus, researching the flow in porous media has become an essential issue in the field of fluid mechanics for engineering.

Classic Darcy's Law was formulated on the basis of numerous experiments. Although it has revealed the fundamental relation of the flow in porous media, its validity is limited for laminar flows at low Reynolds numbers. For non-Darcian flows found in many important natural phenomena, the complexity of flows in porous media has to be addressed. For example, Brinkman (1947) obtained the Darcy-Brinkman equation by adding viscous effect in Darcy's Law, while Joseph et al. (1982) gave a modified Forchheimer's equation by considering inertial effect at high Reynolds number and quadratic drag on the flow in porous media based on previous studies. Vafai and Tien (1981, 1982), and Hsu and Cheng (1990) put forward the Brinkman-Forchheimer equation by combining the above effects, which was gradually improved to be widely applied in generalised Navier-Stokes equation. Plenty of studies confirmed the applicability of generalised Navier-Stokes set. Thus, the foundation was established for the incompressible viscous flow in porous media.

A variety of numerical methods, such as finite difference method (FDM), finite volume method (FVM), finite element method (FEM), were proposed for solving the generalised Navier-Stokes equation. The space and time conservation element and solution element (CE/SE) method, first proposed by Chang (1995), is a new high accurate computational scheme. Wang et al. (2008) proposed an updated CE/SE method. The unique characteristics of the $\mathrm{CE} / \mathrm{SE}$ method is that it unifies space and time, and treats them as an entirety, which is based on the global and local flux in a space-time domain by introducing CEs/SEs. The $\mathrm{CE} / \mathrm{SE}$ method derives a numerical scheme based on Taylor expansion. The accuracy depends on the order of Taylor expansion. The CE/SE method treats both the variables in the flow field and their space derivatives as independent variables. The merits of CE/SE method include its clear physical conception, simple numerical scheme and high computational accuracy.

The CE/SE method has been widely applied in compressible (Wang et al., 2008; Zhang and Wang, 2009) and incompressible multiphase flows by using the preconditioned method (Yang et al., 2012; Yang and Zhang, 2013), as well as poromechanical problems (Yang et al., 2014) governed by hyperbolic equations. Yang et al. (2010) applied the above method to incompressible multiphase flows in porous media, including the contact interface of dual porous medium, lid-driven cavity flows, dual layer lid-driven cavity flow, which expanded the CE/SE method to the field of fluid mechanics in porous media.

This article briefly introduced the high-accuracy CE/SE scheme and the preconditioned method, and simulated unsteady incompressible flows past a square. Pressures and lift coefficients around the cylinder were calculated and compared with other numerical and experimental results. This paper also proposed a model of unsteady incompressible flow around a square porous cylinder, which is associated with the filter of waste water treatment equipment. The vorticity structure and its impact factors were analysed.

Figure 1 Construction of CE/SE method, (a) SE (b) CE

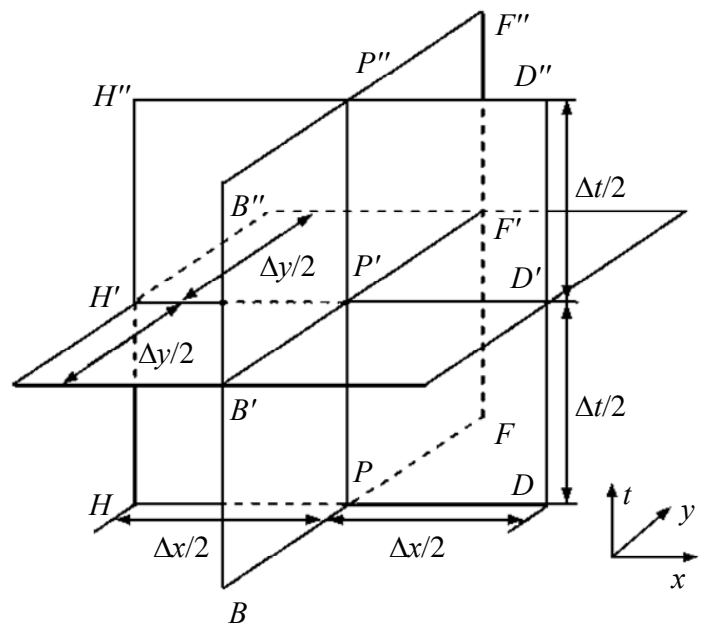

(a)

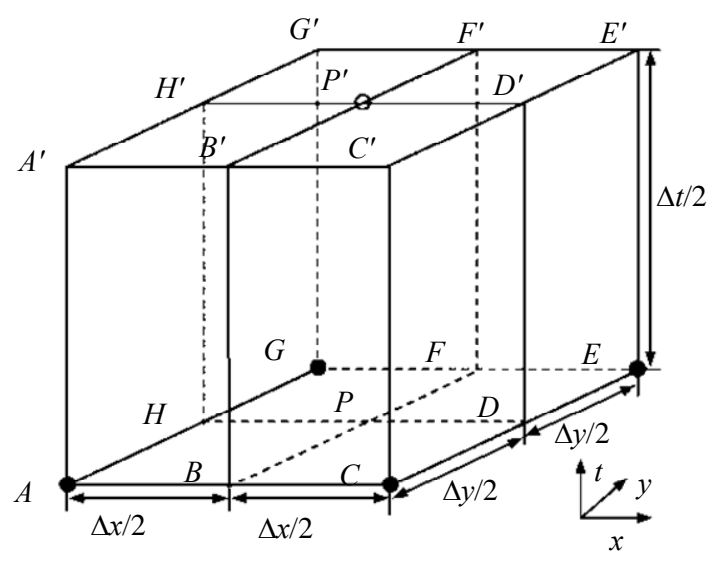

(b)

\section{CE/SE method and governing equation}

Wang et al. (2008) proposed an updated CE/SE scheme based on a hexahedron mesh. As shown in Figure 1, every $\mathrm{CE}$ is corresponding with one SE. The relation between the independent physical variables and their derivatives are derived by the continuity condition of flow variables on a staggered grid. The detailed CE/SE method was referred in 
the literature (Yang and Zhang, 2013). The generalised N-S equation set (Yang et al., 2012) was applied for flows in porous media. The Brinkman and Forchheimer effects were considered in the flow mechanism. The preconditioned procedure (Yang and Zhang, 2013) was applied for transforming the parabolic equations, which govern incompressible viscous flows in porous media, into the hyperbolic ones, which can be directly solved by CE/SE method. A staggered mesh was applied for the calculations in order to avoid a pressure shift phenomenon.

\section{Results and discussion}

The computational domain was constructed for incompressible viscous flows around a square cylinder, as shown in Figure 2. The computational domain covers grid point of $602 \times 162$, and the space and time interval are 0.1 and 0.005 , respectively. The initial and boundary conditions were referred in the literature (Davis and Moore, 1984; Li and Humphrey, 1995). The no-slip boundary conditions were set at the impermeable cylinder surface. The non-dimensional parameters of $\mathrm{D}, \mathrm{L}$ and $\mathrm{H}$ are defined in the glossary.

For the model evaluation, the benchmark problem (Li and Humphrey, 1995) of flow past an impermeable cylinder was simulated. Figure 3 depicted (a) streamlines and (b) vorticity of flows past an impermeable square cylinder at $\operatorname{Re}=100$. As shown in Figure 4(b), there existed a string of staggering vortex, periodically rotating in opposite directions, after flows pass the prism (Pavlov et al., 2000) known as Karman Vortex Street. For further evaluation of the CE/SE method, the coefficient of drag and lift force were analysed. The coefficient of drag force and the lift force as well as Strouhal number were calculated. As shown in Table 1, the CE/SE calculated coefficients corresponded well with those from experiments.

Table 1 Coefficients of flow past an impermeable square cylinder

\begin{tabular}{lcccc}
\hline & $R e$ & $C D$ & $C_{L, r m s}$ & $S t$ \\
\hline CE/SE method & 100 & 1.461 & 0.154 & 0.133 \\
$\begin{array}{l}\text { Experimental (Davis and } \\
\text { Moore, 1984) }\end{array}$ & 100 & 1.66 & 0.18 & 0.164 \\
$\begin{array}{l}\text { Experimental (Okajima, } \\
\text { 1982) }\end{array}$ & 100 & - & - & 0.135 \\
$\begin{array}{l}\text { Numerical (Wan and } \\
\text { Patnaik, 2002) }\end{array}$ & 100 & 1.52 & 0.148 & 0.153 \\
$\begin{array}{l}\text { Numerical (Li and } \\
\text { Humphrey, 1995) }\end{array}$ & 100 & 1.75 & - & 0.141 \\
$\begin{array}{l}\text { Numerical (Pavlov et al., } \\
\text { 2000) }\end{array}$ & 100 & 1.51 & 0.137 & 0.15 \\
\hline
\end{tabular}

Figure 2 Computational domain for flow past a square cylinder

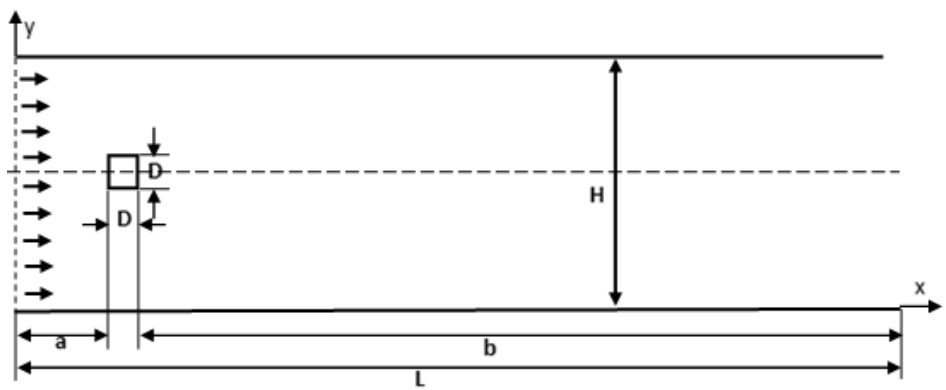

Figure 3 Distribution of streamline and vorticity past a square cylinder for $\operatorname{Re}=100$

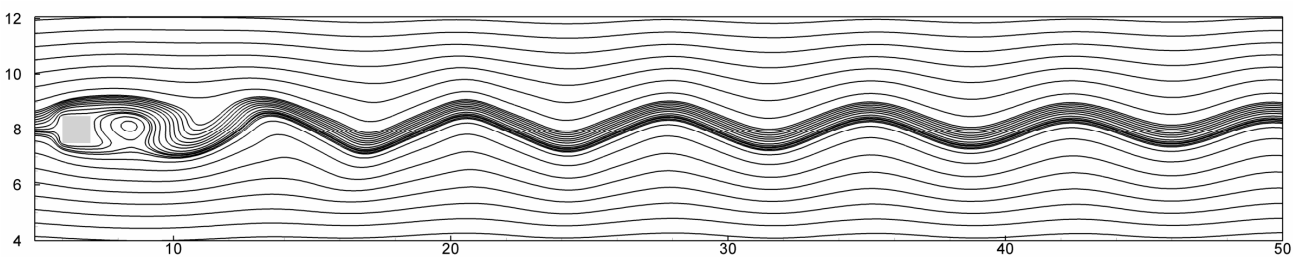

(a)

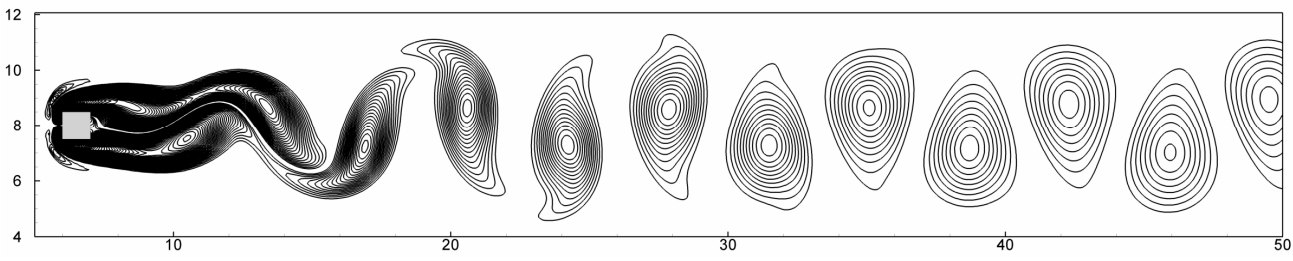

(b) 
Figure 4 Streamlines of flow past (a) impermeable and (b) permeable cylinder for $\mathrm{Re}=100$

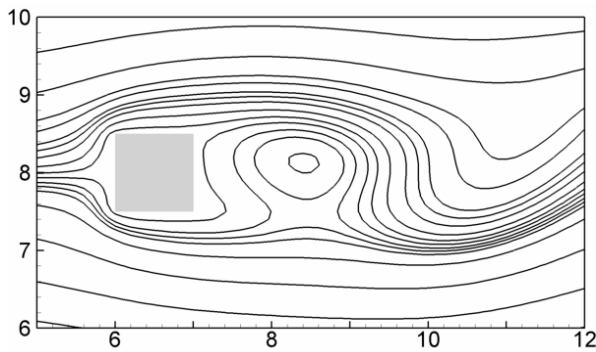

(a)

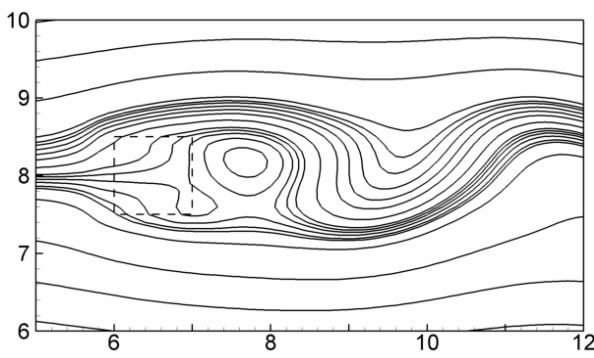

(b)
Figure 4 depicted streamlines around the (a) impermeable and (b) permeable cylinder. The main flow structures were somewhat similar for two specific cases. In case of flow past the permeable cylinder with porosity of 0.1 and Darcy number of $10^{-4}$, the strength of main vortex decreased and the shear layer become weak, compared to the impermeable case. The main reason is that the flow energy attenuated due to the drag, friction from the porous matrix and a decoupled phase movement of the solid and the liquid phase (Yang et al., 2015). It noted that the Darcy number was referred to Yang et al. (2012).

Figures 5 and 6 showed the periodic structure of streamlines and vorticities for flows past the permeable square cylinder with porosity of 0.1 and Darcy number of $10^{-4}$. As depicted in Figure 5, as Reynolds number ( $\left.\mathrm{Re}\right)$ increased, the strength of the main vortex increased, and the shear layer become thinner. For $\mathrm{Re}=50$, two main vortex happened. As Reynolds number increased, the flow fully developed, and such two vortices emerged as a main vortex. The periodic wave happened when the Reynolds number reached 100. As Reynolds number increased, the wave length decreased, and the wave frequency increased.

Figure 5 Streamlines of flow past a permeable square cylinder for (a) $\operatorname{Re}=50$ (b) $\operatorname{Re}=100$ (c) $\operatorname{Re}=300$ (d) $\operatorname{Re}=1,000$ at $\mathrm{Da}=10^{-4}$

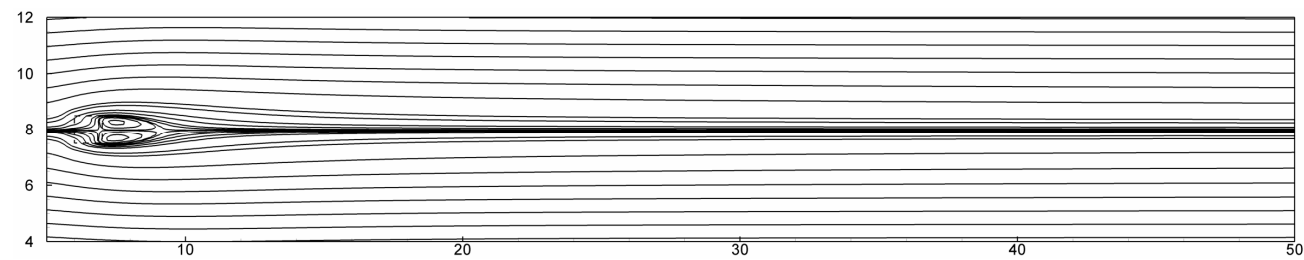

(a)

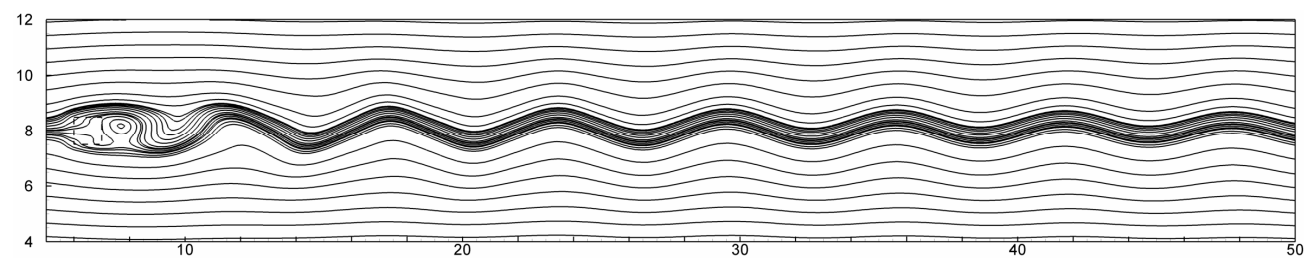

(b)

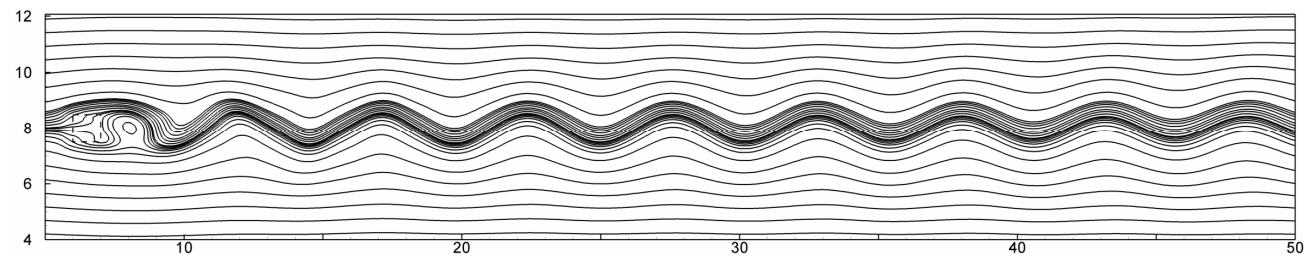

(c)

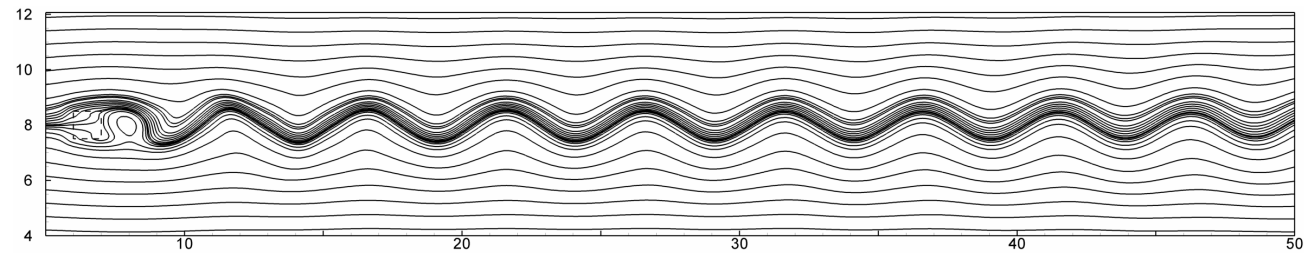

(d) 
Figure 6 Vorticity distribution of flow past a permeable square cylinder at (a) $\operatorname{Re}=50$ (b) $\operatorname{Re}=100$ (c) $\operatorname{Re}=300$ (d) $\operatorname{Re}=1,000$ for $\mathrm{Da}=10^{-4}$

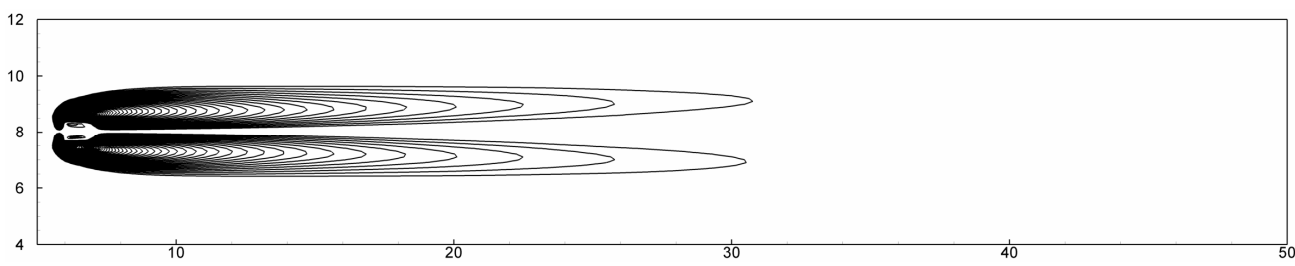

(a)

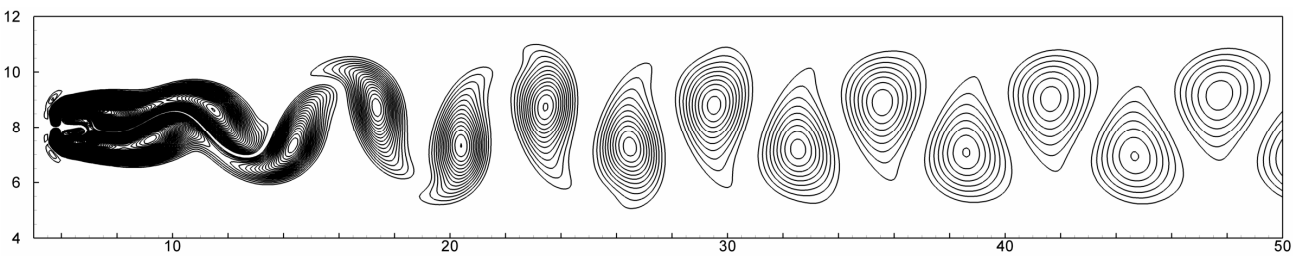

(b)

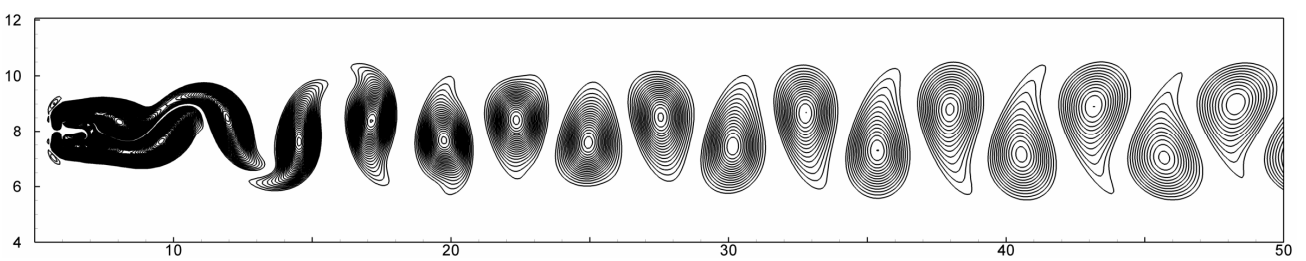

(c)

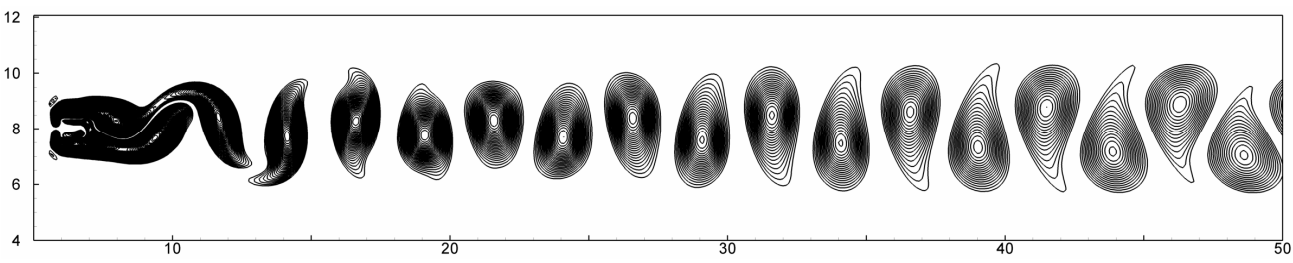

(d)

Figure 7 Evolution of the lift coefficient for flows past impermeable and permeable square cylinder

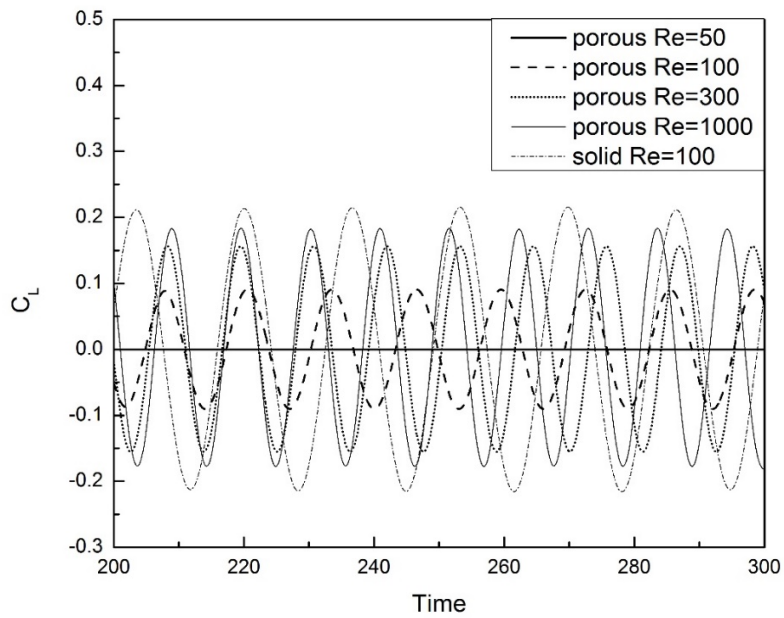

Note: Porous denotes permeable cylinder, while solid presents the impermeable one.
Figure 8 Evolution of the drag coefficient for flows past impermeable and permeable square cylinder

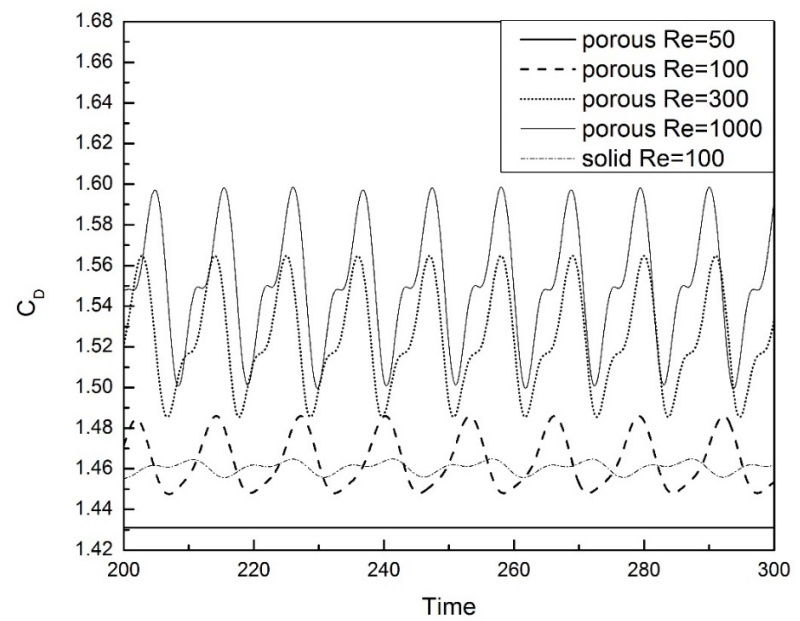

Note: Porous denotes permeable cylinder, while solid presents the impermeable one. 
Figure 9 Streamlines of flow past a permeable square cylinder for $\mathrm{Re}=100, \mathrm{Da}=10^{-4}$ at porosity of 0.3

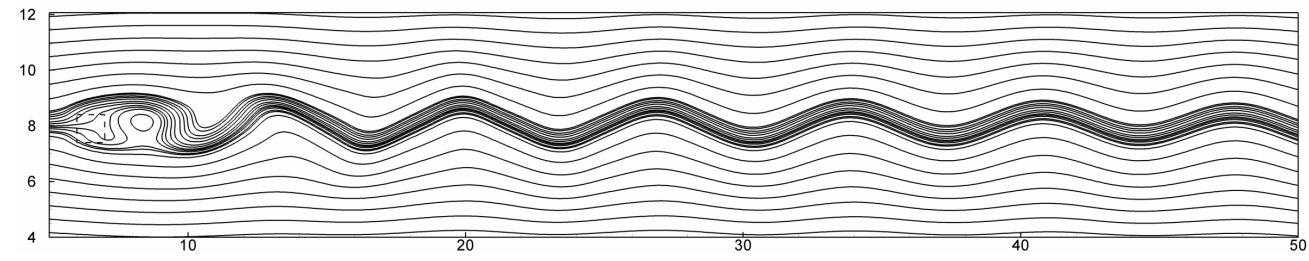

Figure 10 Vorticity distribution of flow past a permeable square cylinder at $\mathrm{Re}=100, \mathrm{Da}=10^{-4}$ for porosity of 0.3

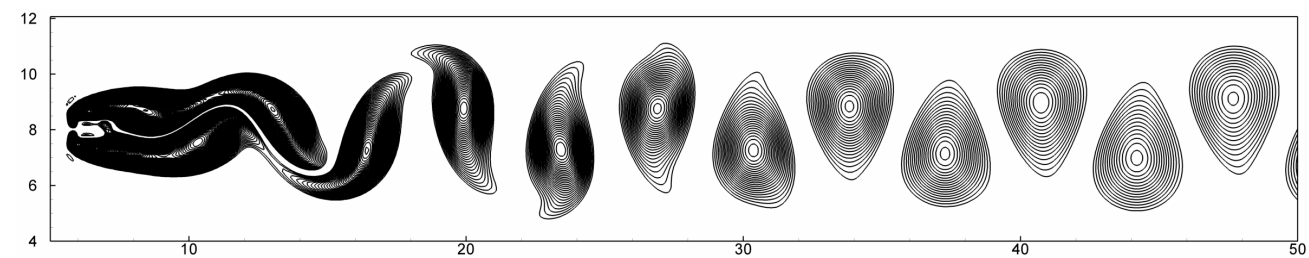

Figure 6 demonstrated the distribution of the vortex shedding. As Reynolds number increased, the numbers of vorticities increased, and the frequency of vortex shedding increased. When Reynolds number reached 100, the periodic wave appeared. Comparing the distribution of the streamline with the corresponding voticity, it indicated that he vortex has significant role in the flow structure near the side face of the cylinder. Table 2 summarised the root-mean-square lift coefficient, mean drag coefficient and the Strouhal number. Given the porosity and the Darcy number, the Reynolds number dominated such parameters. Figures 7 and 8 showed the evolution of lift and drag coefficients, respectively. As the Reynolds number increased, the magnitude of drag and lift coefficient increased, and the wave period of the two coefficients decreased. For the impermeable cylinder at $\mathrm{Re}=100$, the magnitude of drag coefficient decreased, while the magnitude of lift coefficient increased, compared to the permeable one. Porous media remarkably affected on the drag coefficients.

Table 2 Coefficients of flow past permeable and impremeable square cylinders

\begin{tabular}{lcccc}
\hline & $R e$ & $C D$ & $C_{L, r m s}$ & $S t$ \\
\hline Impermeable & 100 & 1.461 & 0.154 & 0.133 \\
& 50 & 1.431 & 0 & \\
Porous media & 100 & 1.464 & 0.067 & 0.167 \\
(porosity 0.1) & 300 & 1.524 & 0.116 & 0.182 \\
& 1000 & 1.551 & 0.135 & 0.2 \\
Porous media & 100 & 1.464 & 0.056 & 0.135 \\
(porosity 0.3) & & & & \\
\hline
\end{tabular}

Figures 9 and 10 demonstrated the structure of streamlines and periodic vorticities for flows past the permeable square cylinder in terms of porosity 0.3 and Darcy number $10^{-4}$ and Reynolds number 100. Compared with Figure 5(b), as porosity increased, the shear layer enhanced. The strength of vortex was elevated for porosity of 0.3 .
Figure 11 Effect of porosity on lift coefficient

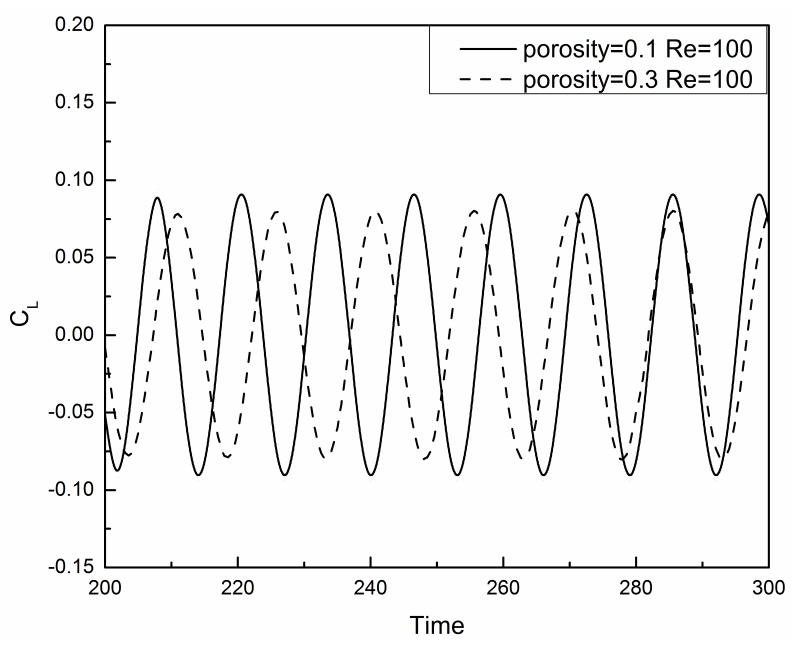

Figure 12 Effect of porosity on drag coefficient

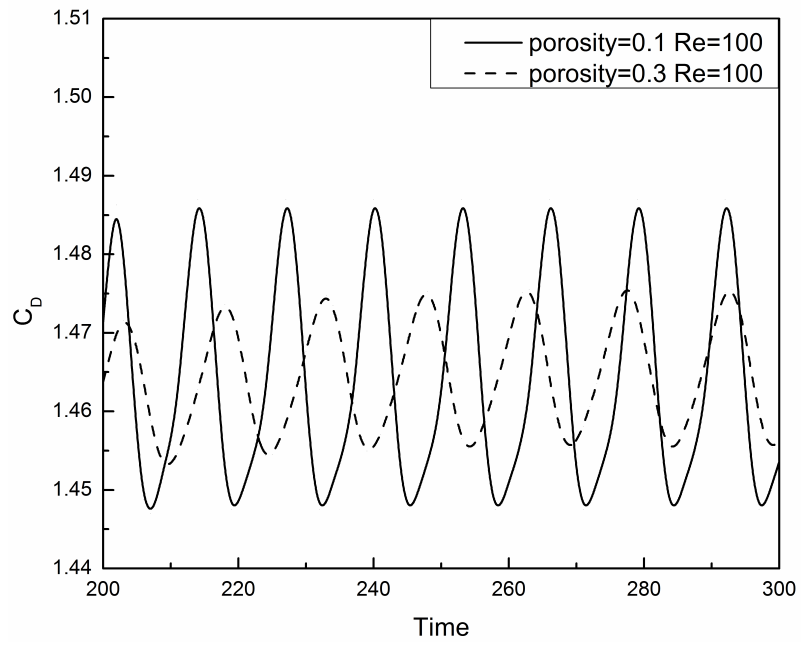

Figures 11 and 12 depicted lift and drag coefficients as function of time for porosity of 0.3 given $\mathrm{Re}=100$ and $\mathrm{Da}=10^{-4}$. As the porosity increased, the magnitude of drag 
and lift coefficient decreased. There are spatially correlated phase lags controlled by the porosity in homogeneous media.

\section{Summary}

Numerical analysis was performed for periodic structures of the streamline and vorticity for flows past a square cylinder. Given the porosity and Darcy number, as Reynolds number increased, the frequency of the vortex shedding increased. As the Reynolds number decreased, the magnitude of drag and lift coefficient decreased, and the wave period of the two coefficients increased. Compared to the permeable one, for the impermeable cylinder at $\mathrm{Re}=100$, the magnitude of drag coefficient decreased, while the magnitude of lift coefficient increased. The vortex has significant role in the flow structure near the side face of the cylinder. Porous media remarkably affected on the flow pattern, drag and lift coefficients.

\section{Acknowledgements}

This work was mainly supported by National '973' Project under Grant No. (2011CB707303) and Institute of Crustal Dynamics (No. ZDL201321). The corresponding author was supported by Technology Foundation for Selected Overseas Chinese Scholar, Ministry of Personnel of China (No. 2014240). The anonymous reviewers have given significant comments and suggestions, which improved this manuscript.

\section{References}

Brinkman, H.C. (1947) 'A calculation of the viscous force exerted by a flowing fluid on a dense swarm of particles', Applied Scientific Research, Vol. 1, pp.27-34.

Chang, S.C. (1995) 'The method of space-time conservation element and solution element - a new approach for solving the Navier-Stokes and Euler equations', Journal of Computational Physics, Vol. 119, pp.295-324.

Davis, R.W. and Moore, E.F. (1984) 'A numerical-experimental study of confined flow around rectangular cylinders', Physics of Fluids, Vol. 27, No. 1, pp.46-59.

Hsu, C.T. and Cheng, P. (1990) 'Thermal dispersion in a porous medium', International Journal of Heat and Mass Transfer, Vol. 33, pp.1587-1597.

Joseph, D.D., Nield, D.A. and Papanicolaou, G. (1982) 'Nonlinear equation governing flow in a saturated porous medium', Water Resources Research, Vol. 18, pp.1049-1052.

Li, G. and Humphrey, J. (1995) 'Numerical modeling of confined flow past a cylinder of square cross-section at various orientations', International Journal of Numerical Methods of Fluids, Vol. 20, No. 11, pp.1215-1236.

Okajima, A. (1982) 'Strouhal numbers of rectangular cylinders', Journal of Fluid Mechanics, Vol. 123, pp.379-398.
Pavlov, A.N., Sazhin, S.S., Fedorenko, R.P. and Heikal, M.R. (2000) 'A conservative finite difference method and its application for the analysis of a transient flow around a square prism', International Journal of Numerical Methods of Heat Fluid Flow, Vol. 10, pp.6-46.

Vafai, K. and Tien, C.L. (1981) 'Boundary and inertia effects on flow and heat transfer in porous media', International Journal of Heat and Mass Transfer, Vol. 24, pp.195-203.

Vafai, K. and Tien, C.L. (1982) 'Boundary and inertial effects on convective mass transfer in porous media', International Journal of Heat and Mass Transfer, Vol. 25, pp.1183-1190.

Wan, D. and Patnaik, B. (2002) 'Discrete singular convolution-finite subdomain method for the solution of incompressible viscous flows', Journal of Computational Physics, Vol. 180, pp.229-255.

Wang, J.T., Liu, K.X. and Zhang, D.L. (2008) 'An improved CE/SE scheme for multi-material elastic-plastic flows and its application', Computers \& Fluids, Vol. 38, No. 3, pp.544-551.

Yang, D.X. and Zhang, D.L. (2013) 'An improved CE/SE scheme for incompressible multiphase flows and its applications', Progress in Computational Fluid Dynamics, Vol. 13, No. 5, pp.285-295.

Yang, D.X., Li, Q. and Wang, S. (2014) 'Numerical analysis of propagation of pore pressure waves in compressible fluid saturated porous media', Rock and Soil Mechanics, Vol. 35, No. 7, pp.2047-2056.

Yang, D.X., Li, Q. and Zhang, L.Z. (2015) 'Propagation of pore pressure diffusion waves in saturated porous media', Journal of Applied Physics, Vol. 117, Nos. 1-5, pp.134902, DOI: 10.1063/1.4916805.

Yang, D.X., Xue, Z.Q. and Simon, A.M. (2012) 'Analysis of momentum transfer in a lid-driven cavity containing a Brinkman-Forchheimer medium', Transport in Porous Media, Vol. 92, No. 1, pp.101-118.

Yang, D.X., Zhang, D.L., Zeng, R.S. et al. (2010) 'Calculation of multiphase flows in porous media based on CE/SE method', Chinese Journal Geophysics, Vol. 53, No. 1, pp.189-196.

Zhang, D.L. and Wang, J.T. (2009) 'An improved CE/SE scheme and its application', Chinese Journal of Computational Physics, Vol. 26, No. 2, pp.211-220.

\section{Glossary}

$$
\begin{aligned}
& C_{d}=F_{d} / \frac{1}{2} \rho U^{2} D: \text { drag coefficient } \\
& C_{l}=F_{l} / \frac{1}{2} \rho U^{2} D: \text { lift coefficient } \\
& \mathrm{C}_{\mathrm{l}, \mathrm{rms}}: \text { mean drag coefficient } \\
& \mathrm{St}=f D / U: \text { Strouhal number } \\
& f: \text { frequency of vortex shedding } \\
& \mathrm{L} \times \mathrm{H}=60 \times 16 \\
& \mathrm{a}=6 \text { and } \mathrm{b}=53.0 \\
& \mathrm{D}=1
\end{aligned}
$$

